\title{
A New Hybrid Optimization Algorithm Framework to Solve Constrained Optimization Problem
}

\author{
Huang Zhangcan ${ }^{1}$ and Cheng $\mathrm{Hao}^{2}$ \\ ${ }^{1}$ School of Science, Wuhan University of Technology, Wuhan 430070, China \\ ${ }^{2}$ School of Mechanical and Electrical Engineering, Wuhan University of Technology, \\ Wuhan 430070, China \\ \{huangzc, h. cheng\}@whut .edu.cn
}

\begin{abstract}
Evolutionary Computation made great success from the theory of natural selection devised by Charles Darwin. It was a process of randomly searching but not emphasizing each individuals respective functions. This paper proposed a hybrid optimization algorithm framework trying to incorporate natural selection and survival of the fittest and birds of a feather flock together. Aiming at balancing search results and search speed, we adopted the search strategy to classify the individuals by their fitness. Individuals classification differentiated respective function in search process, thats the excellent individuals mine the local optimal solution and others explore the search domain to find new local optimal solution. Experimental findings support the theoretical basis of the proposed framework.
\end{abstract}

\section{Introduction}

Solving a constrained optimization problem with inequality, upper bound, and lower bound constraints usually includes mathematical model building and algorithm designing. Many methods have been posed from Newton Method, Linear Search to Evolutionary Computation 1], which has respective characteristics. Evolutionary computation from the theory of Natural Selection and Survival of the Fittest has long been accepted as a powerful search tool in both academia and industry, with numerous applications in varies science and engineering problem. The Darwinian-type evolutionary computation uses mutation (an asexual reproduction with variation), crossover (a recombination or sexual reproduction) and selection (survival of the fitness). These operators are simple to execute and domain-independent 2]. Michalski employs machine learning to generate new populations, called Learnable Evolutionary Model, briefly LEM. LEM integrated machine learning and evolutionary computation, which focused on why certain individuals are superior to others 3. SUN employs similartaxis and dissimilation instead of crossover and mutation operators in Mind Evolutionary Computation, briefly MEC 4. For low efficiency of conventional methods to dealing with highly complex problems, more methods like LEM, MEC trying to

Y. Shi et al. (Eds.): ICCS 2007, Part IV, LNCS 4490, pp. 1005 1012, 2007.

(C) Springer-Verlag Berlin Heidelberg 2007 
emphasis individuals characteristics. The novel idea proposed in this research is to incorporate "birds of a feather flock together", a universal rule of human activity and "natural selection and survival of the fittest", Darwinian-type EC together to arrange each individual respective functions.

One of the most critical of these features is perhaps that how to build effective algorithm framework to solve more and more complex mathematical model. Jim Gray believes a good long-range goal should have five key properties: Understandable, Challenging, Useful, Testable, Incremental [5]. According to these rules, we try to build a hybrid optimization algorithm framework which focus on two goals. One is to unify the optimization problems into a framework and then use the framework to guide the mathematical models building, the other is to incorporate different algorithms search kernel.

Subsequent sections of this paper deal with this issue of usage of the framework to design algorithm to solve constrained optimization problem. The organization of the paper is as follows: In Section 2 defines the general constrained optimization problems. Section 3 presents the framework and its major functional aspects. Some experimental results and discussions are presented in Section 4, while conclusions are drawn in Section 5.

\section{Statement of the Problem}

The general constrained optimization problem can be defined as follows:

$$
\begin{aligned}
& \text { minimize } f(\boldsymbol{x}) \\
& \text { subject to } g_{j}(\boldsymbol{x}) \leq 0, j=1, \ldots, J \\
& h_{m}(\boldsymbol{x})=0, m=1, \ldots, M \\
& x_{i}^{L} \leq x_{i} \leq x_{i}^{U}, i=1, \ldots, n
\end{aligned}
$$

where $f(\boldsymbol{x})$ is an objective function, $\boldsymbol{x}$ is the vector of decision variables $\boldsymbol{x}=$ $\left[x_{1}, x_{2}, \ldots, x_{n}\right], J$ is the number of inequality constraints, and $M$ is the number of equality constraints (objective function, constraints could be linear or nonlinear).

$x_{i}^{L}$ and $x_{i}^{U}$ are the upper bound and the lower bound of $x_{i}$, respectively. Denote with $F$ to the feasible region and with $S$ to search space. The feasible solutions exist in $F \subseteq S$.

\section{Description of Hybrid Optimization Algorithm Framework}

In our previous work, multi-population adaptive-gathering evolutionary algorithm 6] using domains of attraction to separate and gather the initial population automatically to deal with function optimization, which divide the entire search space of objective function into several domains. In another paper, neighborhood exploring evolutionary strategy 7 consists of assigning neighborhood of different size to different solution to deal with multi-objective optimization problem. The population classification and neighborhood assignment behind these ideas are also the foundation of the Hybrid Optimization Algorithm Framework as our forward work. 


\subsection{Population Classification}

The first critical step in the process of the framework is to calculate the individuals' fitness at every generation and to sort the population by their fitness. The individuals are selected randomly and they have their performance, better or worse. Most Evolutionary Computation algorithms work with population of fixed size keeping at least the best individual always in the current population 1. No signs indicate part of the worse individuals remain in next population will hinder optimum seeking. If we ignore some worse individuals we lost the diversity of the population especially in multi-modal objective function space. Based on this idea, we intend to classify the population into several levels in the current population. We assign individuals in each level with different tasks to generate new offspring. The individuals in the next population will be selected from each level. Hence, we arrange each individual respective function and at the same time keep the diversity of the population.

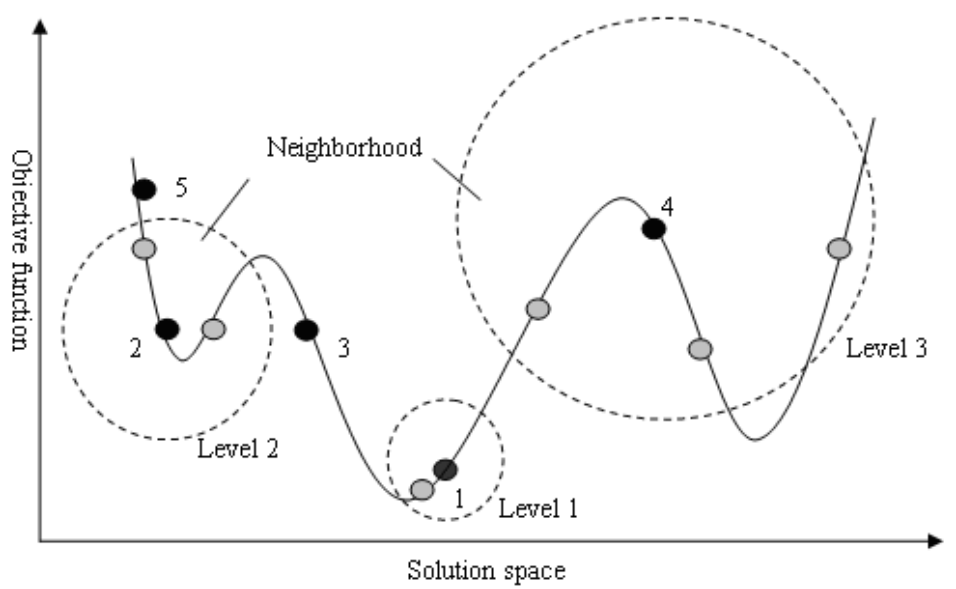

Fig. 1. Illustration of generating new offspring using $(1+\lambda)$-ES. The black dots denote father individuals and the grey dots denote offspring.

Figure 1illustrates an example of such classification. There are five solutions in the solution space that are classified into three group, 1,2,3,4,5according to the individual's fitness. We assign each solution a level, for instance, in Figure 1, solution 1 in level 1 , solution 2, 3 in level 2, solution 4, 5 in level 3.

\subsection{Neighborhood Assignment}

After population classification, the next step is to decide the neighborhood for every solution in the population. The neighborhood is designed in the decision variable space and the actual shape of the neighborhood can be various. 
In this way, we can assign neighborhood to each level. The solutions with lower levels have small sized neighborhood and vice verse. The solutions in the same level have same sized neighborhood. For convenience, we use a circle denote a neighborhood and several different neighborhoods are showed in Figure 1.

\section{$3.3 \quad(1+\lambda)$-ES (Evolutionary Strategy) Selection}

$(1+\lambda)$-ES is an efficient selection method in evolutionary strategy, which generates $\lambda$ offspring using the information 1 individual in the population [2]. The $(1+\lambda)$-ES can be used to generate new offspring. In this case, the individual in the level 1 uses $(1+1)$-ES, level 2 uses $(1+2)$-ES and level 3 uses $(1+3)$-ES.

\subsection{Hybrid Optimization Algorithm Framework}

Evolutionary algorithms deserve a special mention as powerful global optimizers, but which not emphasis on each individuals' respective function. It is a main difficult to balance search speed and result.

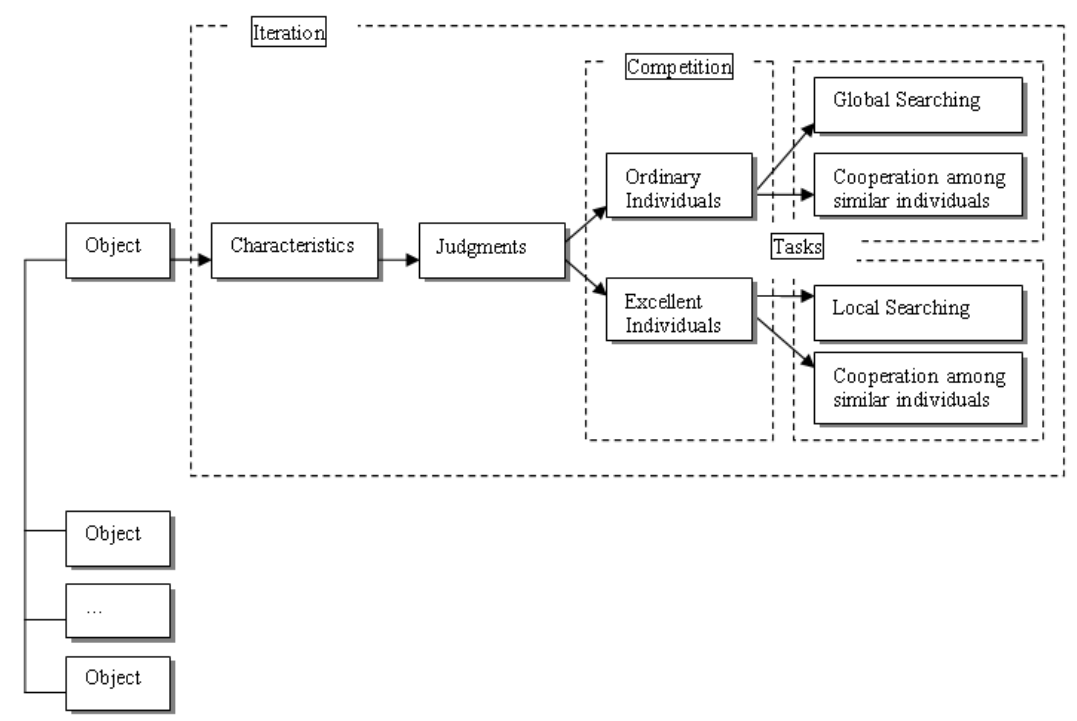

Fig. 2. The proposed optimization algorithm framework

We try to arrange the task to each individual from the theory of Birds of a Feather Flock Together. No matter how the optimization objects and objective changed, the characters of objectives on optimization objects have two respects. Firstly, capability similarity, that's ordinary individuals gather to excellent individuals. Secondly, structure similarity, that's those individuals with similar capability have similar structure in some kinds. 
The Hybrid Optimization Algorithm Framework is based from randomly population search. To explain how the framework works, we consider an optimization problem as the object and the object functions as their characteristics. By judging each characteristic, we classify the population into different level. By competition among the called ordinary individuals and excellent individuals, we arrange their respective tasks. That is by cooperation among ordinary individuals to explore hopefully domain with global searching and excellent individuals to mine the local minima with local searching (see Figure 2).

\subsection{Using the Framework to Design Algorithm}

Under the guidance of the framework, we can design algorithms to solve constrained optimization problems. The process can be described as following:

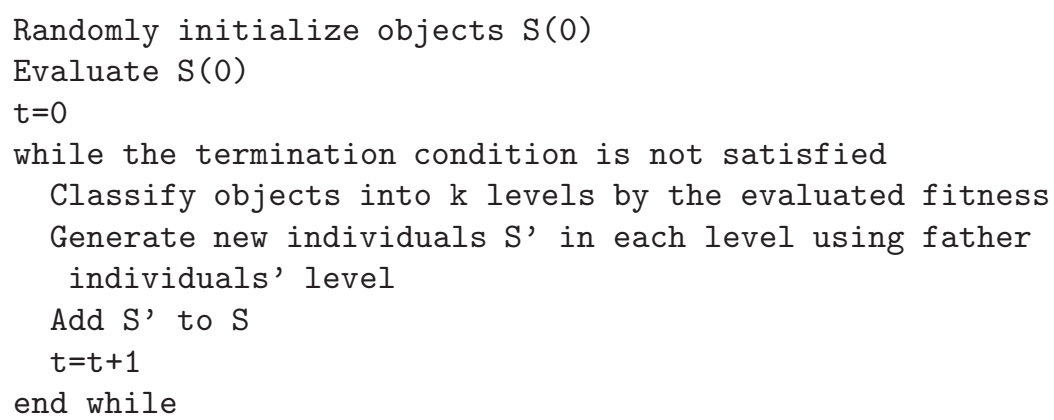

How to classify objects into k levels according to the problem's complex and the human personal experience. We consider individuals' similarity in their characteristic.

$$
\begin{array}{r}
f\left(x_{\text {min }}^{*}\right)+\frac{p}{k} L<f\left(x_{i}\right) \leq f\left(x_{\text {min }}^{*}\right)+\frac{p+1}{k} L \\
x_{i} \in p \quad \text { level }, p=1,2, \ldots, k-1
\end{array}
$$

Where $x_{i} \in S, f\left(x_{i}\right)$ is the fitness of each individual (see Equation (3),(4)) and $\mathrm{L}$ is the distance between two individuals (see Equation (5)).

$$
\begin{gathered}
f\left(x_{\max }^{*}\right)=\max f\left(x_{i}\right) \\
f\left(x_{\min }^{*}\right)=\min f\left(x_{i}\right) \\
L=\max f\left(x_{i}\right)-\min f\left(x_{i}\right)
\end{gathered}
$$

\section{Statement of the Problem}

Examples mentioned in this chapter are representatives of different kinds of functions. Example 1 is cited from Ref. [8, which has been known that when $n=2$, the function has 720 local optimal solutions, 18 of which are global optimal solutions. Example 2 is cited from Ref. [9], which is nonlinear and its 
global modal is unknown. We adopted real code and the experiment parameters are: population size $N=20$, initialized radius $r_{0}=c 2^{p-1}$ and level $k=4$. Here, $p$ is the individual's level, $c$ is a constant, which is set by domain radius.

Example 1: n-demension Shubert function

$$
\begin{array}{r}
\min \quad f\left(x_{1}, x_{2}, \ldots, x_{n}\right)=\prod_{i=1}^{n} \sum_{j=1}^{5} j \cos \left((j+1) x_{i}+j\right) \\
x_{1} \in[-10,10], i=1,2, \ldots, n
\end{array}
$$

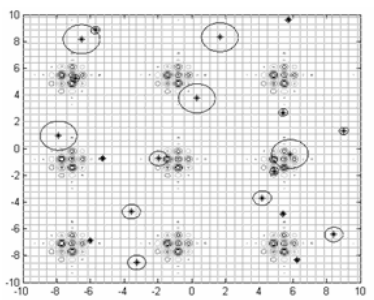

(a)

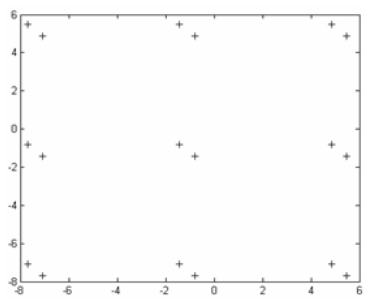

(b)

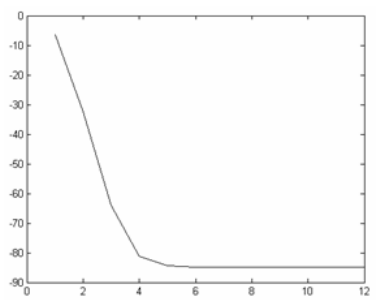

(c)

Fig. 3. (a)Distribution of Initial population and its levels. (b)distribution of the 12th generation. (c)Curve of convergence.

Table 1. Results observed under different contractive ratio $c$

\begin{tabular}{ccccc}
\hline$c$ & $\begin{array}{c}\text { Average } \\
\text { modals found }\end{array}$ & $\begin{array}{c}\text { Running } \\
\text { times }\end{array}$ & $\begin{array}{c}\text { Times } \\
\text { approaching modals generations running }\end{array}$ \\
\hline 1 & 3 & 10 & 5 & 730 \\
0.9 & 6 & 10 & 9 & 88 \\
0.8 & 5 & 10 & 10 & 46 \\
0.7 & 5 & 10 & 10 & 30 \\
0.6 & 4 & 10 & 10 & 23 \\
0.5 & 3 & 10 & 10 & 17 \\
0.4 & 2 & 10 & 10 & 14 \\
0.3 & 1 & 10 & 10 & 11 \\
\hline
\end{tabular}

Example 1 is a high-dimension function, which have 18 global optimal solutions (see Figure 31). At 12th generation the algorithm obtained global solutions.

To test the effects of the parameters on the algorithm, we varied the ratio $c$ on Shubert function. The result can be viewed in Table 1. When $c=1$, the searching process is global searching, not decreasing the convergence speed but found few modals. When $c=0.9,0.8,0.7$, more modals were found and speed up the convergence speed. When $c=0.6,0.5,0.4,0.3$, modals can be found in each run quickly, but more modals were failed to find. From the above result, we can see for ordinary optimization problems, the $c$ should be in 0.6 to 1 . From the point of searching, the smaller searching domain is the easier to converge to 
local minima. When holding a larger $c$ and in a larger searching domain, it is more easy to find global minima. When decrease $c$, the algorithm has a quick convergence speed but probably the result is local minima.

Example 2: Bump Function

$$
\begin{array}{r}
f(X)=-\left|\frac{\sum_{i=1}^{n} \cos ^{4}\left(x_{i}\right)-2 \prod_{i=1}^{n} \cos ^{2}\left(x_{i}\right)}{\sqrt{\sum_{i=1}^{n} i x_{i}^{2}}}\right| \\
\prod_{i=1}^{n} x_{i} \geq 0.75, \sum_{i=1}^{n} x_{i} \leq 0.75 n, 0 \leq x_{i} \leq 10, i=1,2, \ldots n
\end{array}
$$

Table 2. Results of bump function in 20, 50, 100 dimension

\begin{tabular}{lccc}
\hline Dimensions & 20 & 50 & 100 \\
\hline Framework & -0.803615 & -0.835196 & -0.842786 \\
Ref. [8], [9] & -0.8035 & -0.8352620 & -0.8410 \\
\hline
\end{tabular}

Example 2 is a high-dimension bump function, which is very difficult to solve. Generally, we get satisfied results in 20, 50, 100 dimensions (see Table 2).

\section{Conclusion and Future Works}

In this study, natural selection and survival of the fittest, birds of a feather flock together, are incorporated into a hybrid optimization algorithm framework by arranging each individual's task.

With regards to algorithm from the framework, further work is necessary to improve the method of individuals' classification and local speed search. Also, the way of deciding the proper contractive ratio $c$ should be devised. The used $\operatorname{method} c$ is set to 0.7 , but there may be a more appropriate value to enhance the quality of convergence. It may be desirable to devise a guide to choose the proper value of $c$. To test the high dimensional bump function is a remarkable performance, we just adopted simply acceleration and many excellent methods, like greedy algorithm, are value to test in the framework, how to make them cooperate perfectly is our future work.

\section{References}

1. Christian Blum, Andrea Roli: Metaheuristics in Combinatorial Optimization: Overview and Conceptual Comparison. ACM Computing Surveys, 35 (3). ACM, Inc., New York (2003) 268-308

2. Zbigniew Michalewicz, David B.Fogel: How to Solve it: Modern Heuristics. SpringerVerlag Berlin Heidelberg.(2000) 
3. Ryszard S.Michalski: Learnable Evolutionary Model: Evolutionary Processes Guided by Machine Learning. Machine Learning. 38 (1-2). Springer, Netherlands (2000) 9-40

4. Sun Chengyi, Zhou Xiuling, Wang Wanzhen: Mind Evolutionary Computation and Applications. Journal of Communication and Computer. 1 (1). USA-China Business Review (Journal), Inc., USA (2004) 13-21

5. Jim Gray: What Next? A Dozen Information-Technology Research Goals. Journal of the ACM, 50 (1). ACM, Inc., New York (2003) 41-57

6. Chen Siduo, Huang Zhangcan: Multi-population adaptive-gathering evolutionary algorithm in function optimization. Proceedings of the 2000 Evolutionary Computation Congress, 1. IEEE (2000) 817-821

7. Hu Xiaolin, Carlos A. Coello Coello, Huang Zhangcan: A New Multi-objective Evolutionary Algorithm: Neighborhood Exploring Evolution Strategy. Engineering Optimization, 37. Taylor and Francis Ltd, UK(2005) 351-379

8. Huang Yuzhen, Kang Lishan, Zhou Aimin: Two-Phase Genetic Algorithm Applied in the Optimization of Multi-Modal Function. Wuhan University Journal of Natural Sciences, 8. Wuhan University Journals Press, Wuhan(2003) 259-264

9. Kang Zhuo, Li Yan, Liu Pu, et al: Two Asynchronous Parallel Algorithms for Function Optimization. Wuhan University Journal of Natural Sciences, 48 (1). Wuhan University Journals Press, Wuhan (2002)33-36 